\title{
Comparison of Self-Calibration Methods for Measurement Channels in Respect of a Method of Conversion Functions Interpolation
}

\author{
W. Gawędzki \\ Department of Measurement \& Instrumentation, AGH-University of Science and Technology, \\ al. Mickiewicza 30,30-059 Kraków, Poland, e-mail: waga@agh.edu.pl
}

\begin{abstract}
The paper suggests algorithms enabling self-calibration of measurement channels with linear as well as nonlinear conversion functions. To approximate conversion characteristics, interpolation methods are used. They are widely known; however, the essence of the suggested self-calibration method is the current modernization of interpolating functions parameters due to a suitable procedure. It enables correction of the two essential error components which may appear in the measurement channel by means of evaluating the current value of the real slope of the conversion characteristic as well as of the components describing nonlinearity generated by disturbing factors. The methods of self-calibration for measurement channels cooperating with non-electrical quantities sensors are compared. Interpolation of conversion functions is assumed as linear in intervals and polynomial.
\end{abstract}

Keywords: self-calibration, non-electrical monitoring, sensors, interpolation.

\section{INTRODUCTION}

$\mathrm{M}$ EASURING SYSTEMS operating during prolonged measurements as well as in terms of significant scattering of measurement points require specific procedures of self-diagnostics and analysis of technical state for individual elements of a measurement channel.

Likewise, an analysis of self-calibration methods of the system measurement channels is necessary, independently on the initial calibration of the measurement channel. They facilitate the rendering of independent measurements results from possible changes influenced by parameters of electronic elements. Procedures of self-calibration most frequently refer to the accomplishment of prolonged measurements (monitoring) of non-electrical quantities. Also, they enable either to avoid troublesome initial adjustment of the measurement channels elements, or to use cheaper elements of lower quality, without worsening the accuracy and stability of the whole measurement system [1]. Self-calibration procedures are mostly limited to the electronic parts of measurement systems and consist of current evaluation of present values of the conversion functions parameters. It is necessary to employ calibrating reference elements (depending on the sensor type, e.g. standard voltage sources, standard resistors, voltage dividers etc.). Two elements are necessary for a linear conversion function; more than two for a non-linear one [2], [3]. For a vast majority of cases, selfcalibration procedures do not include sensors, because they would require troublesome and frequently difficult realization, using measured non-electrical quantity standards.

\section{MEASUREMENT CHANNEL SENSITIVITY}

Conversion function of a channel for the measurement of a non-electrical quantity (Fig.1) may be expressed as a composi- tion of a sensor conversion function $f$ and an electronic part of the measurement channel $g$ [2]. For a general case of nonlinear functions $f$ and $g$ :

$$
\begin{gathered}
y=f(x), \\
z=g\left(y, a_{0}, a_{1}, \ldots a_{n}\right),
\end{gathered}
$$

where: $a_{0}, a_{1}, \ldots a_{n}$ - parameters of the conversion channel of the measuring apparatus, which may change due to time, temperature, or other influencing factors. Substituting (1) into (2) we have:

$$
z=g\left(f(x), a_{0}, a_{1}, \ldots a_{n}\right)
$$

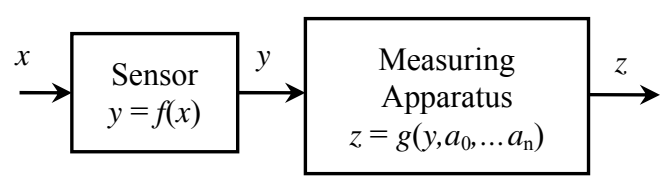

Fig. 1 Measurement channel diagram.

Assuming that sensitivities of the measuring apparatus and the sensor are $S_{A}$ and $S_{S}$ respectively, the measurement channel sensitivity is:

$$
\begin{array}{r}
S=\frac{d z}{d x}=\frac{\partial z}{\partial y} \cdot \frac{d y}{d x}=g^{\prime}(y) \cdot f^{\prime}(x)= \\
S_{A}\left(y, a_{0}, a_{1}, \ldots a_{n}\right) \cdot S_{S}(x)
\end{array} .
$$

The resultant sensitivity of a measurement channel is a product of sensitivities of all its elements. Such approach makes it possible to mould the total measurement channel sensitivity by means of the choice of individual element's sensitivities. 
Let us consider a case when the sensor conversion characteristic is either linear or non-linear, but known and invariable; however, the parameters of the channel conversion function may vary with time or disturbing factors during a prolonged measurement. Let us assume, additionally, that the conversion function of the apparatus $S_{A}\left(y, a_{0}, a_{1} \ldots a_{n}\right)$ may be in a general case non-linear. As a matter of fact, at the stage of manufacturing the apparatus it is possible to fit its parameters so as to eliminate non-linearity errors. However, during the exploitation, particularly within a prolonged working cycle with a significant influence of the disturbing factors, the possibilities for changes in the conversion function parameters and emergence of non-linearity errors have to be taken into account. By means of multipoint calibration [4], [5] (Fig. 2) it is possible to evaluate the present values of the conversion function parameters. The results of measurements between the calibration points are evaluated by means of interpolation: linear, linear in intervals, or with polynomial functions, when interpolation knots are determined by the values of calibrating quantities $y_{0}, y_{1} \ldots y_{n}$, substituting during calibrating procedure the output signal y of the sensor. Depending on the type of the sensor output quantity, as the reference quantities voltage sources, standard resistors, voltage dividers etc. are accepted. Position " $y$ " of the switch $\mathrm{P}$ denotes the function of measurement of the unknown non-electrical quantity $x$.

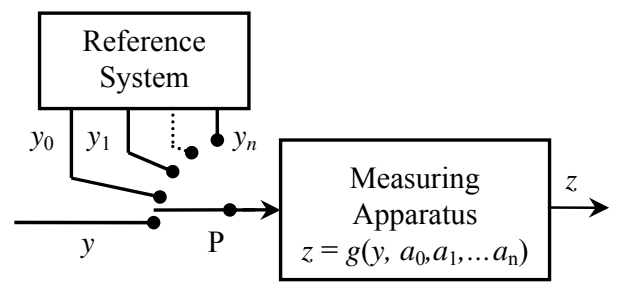

Fig. 2 Schematic diagram of a measurement channel self-calibration.

\section{ALGORITHM OF A CHANNEL SELF-CALIBRATION BY MEANS OF LINEAR IN INTERVALS INTERPOLATION OF THE CONVERSION FUNCTION}

Let's present interpolation of a conversion function of a measuring apparatus with spline functions of 1st order (so called functions linear in intervals) in closed interval $<\alpha$; $\beta>$ for $(n+1)$ calibration points $y_{0}, y_{1}, \ldots y_{n}$, while:

$$
\alpha=y_{0}<y_{1}<\ldots<y_{n-1}<y_{n}=\beta \text {. }
$$

Points $y_{i}$ for $i=0,1, \ldots n \quad(n \geq 1)$ determine a partition of the interval $<\alpha ; \beta>$ to $n$ subintervals, and assuming that this partition is uniform, we have:

$$
y_{i}=y_{0}+i \cdot h \quad h=\frac{\beta-\alpha}{n} \quad i=0,1, \ldots, n .
$$

Let us represent linear in intervals interpolation $\varphi(y)$ of the conversion function of measurement apparatus $g\left(y, a_{0}, a_{1} \ldots a_{n}\right)$ (2):

$$
\varphi_{i}(y)=1 / h \cdot\left[\left(z_{i}-z_{i-1}\right) \cdot y+\left(z_{i-1} y_{i}-z_{i} y_{i-1}\right)\right] \quad i=1, \ldots, n .
$$

It is easy to notice that at the interpolation knots, determining $i$-th interval $\left\langle y_{i-1} ; y_{i}\right\rangle$, the values of interpolating function are:

$$
\begin{aligned}
& \varphi_{i}\left(y_{i-1}\right)=g\left(y_{i-1}\right)=z_{i-1} \\
& \varphi_{i}\left(y_{i}\right)=g\left(y_{i}\right)=z_{i} \quad i=1, \ldots, n
\end{aligned}
$$

Using notations:

$$
\begin{aligned}
& p_{i}=1 / h \cdot\left(z_{i}-z_{i-1}\right) \\
& q_{i}=1 / h \cdot\left(z_{i-1} y_{i}-z_{i} y_{i-1}\right) \quad i=1, \ldots, n
\end{aligned}
$$

we get the final relationship for the interpolating function within the i-th interval:

$$
\varphi_{i}(y)=p_{i} \cdot y+q_{i} \quad i=1, \ldots, n .
$$

For the assigned $n+1$ standard values $y_{0}, y_{1}, \ldots y_{n}$ (Fig. 2) it is necessary to determine $n+1$ corresponding output values $z_{0}$, $z_{1}, \ldots z_{n}$ of the equipment (according to (8)) and then to determine coefficients $p_{i}$ and $q_{i}$ of the interpolating function according to (9).

The application of this type of interpolation is known; however, the essence of the suggested method is the current modernization of the interpolating function parameters $p$ and $q$ due to the application of a suitable self-calibration procedure. It is then possible to correct two essential error components (sensitivity and non-linearity errors), which may appear in the measurement channel, by means of evaluating the current value of the conversion characteristic real slope and the components describing non-linearity generated (or modified) by the disturbing factors in the measurement channel.

\section{SELF-CALIBRATION ALGORITHM USING POLYNOMIAL INTERPOLATION OF THE CONVERSION FUNCTION}

Let us expand the conversion function of the apparatus $g\left(y, a_{0}, a_{1} \ldots a_{n}\right)(2)$ into Taylor series according to the variable $y$ powers within zero neighborhood $\left(a_{0}, a_{1} \ldots a_{n}\right.$ are the function parameters):

$$
z(y)=z(0)+\frac{1}{1 !} \cdot y \cdot z^{\prime}(0)+\frac{1}{2 !} \cdot y^{2} \cdot z^{\prime \prime}(0)+\ldots .
$$

Using notations:

$$
\begin{aligned}
& a_{0}=z(0), \quad a_{1}=\frac{1}{1 !} \cdot z^{\prime}(0), \quad a_{2}=\frac{1}{2 !} z^{\prime \prime}(0), \ldots \quad \text { and } \\
& z_{0}=z\left(y_{0}\right), \quad z_{1}=z\left(y_{1}\right), \quad z_{2}=z\left(y_{2}\right), \ldots
\end{aligned}
$$


and taking them into account in (11) we obtain for $n+1$ knots $y_{0}, y_{1}, \ldots y_{n}$, for which the values of the interpolated function are equal $z_{0}, z_{1}, \ldots z_{n}$, a system of $n+1$ equations with searched coefficients $a_{0}, a_{1}, \ldots a_{n}$ of the conversion function. Using $n+1$ calibration points $(n+1$ knots) and accepting order $n$ of the interpolating polynomial, for an arbitrary spacing of interpolation knots $y_{i}$ it is possible to evaluate the Lagrange interpolating polynomial $\varphi(y)$ :

$$
\varphi(y)=\sum_{i=0}^{n} z_{i} \cdot \frac{\omega_{n}(y)}{\left(y-y_{i}\right) \cdot \omega_{n}^{\prime}\left(y_{i}\right)},
$$

where:

$$
\omega_{n}(y)=\left(y-y_{0}\right)\left(y-y_{1}\right) \ldots\left(y-y_{n}\right)
$$

and $\omega_{n}^{\prime}\left(y_{i}\right)$ is the value of the polynomial $\omega_{n}(y)$ derivative at the point $y_{i}$.

To approximate real conversion function (2) of a measurement apparatus with a polynomial of the $n^{\text {th }}$ order it is necessary to assign $n+1$ standard values $y_{0}, y_{1}, \ldots y_{n}$ (Fig. 2) and to determine $n+1$ corresponding output values $z_{0}, Z_{1}, \ldots z_{n}$. Interpolating polynomial, which enables to determine $z$ value for arbitrary $y$ from among $y_{i}$ and $y_{j}$, is determined on the grounds of (13) and (14).

The essence of the suggested method is the current modernization of the interpolating polynomial parameters due to the application of a suitable self-calibration procedure (Fig. 2).

A similar effect of self-calibration may be achieved also with other interpolating functions. In any case, it is necessary to use reference standard quantities (Fig. 2). Their accuracy and stability will determine self-calibration procedure accuracy and efficiency.

\section{COMPARISON OF SELF-CALIBRATION ALGORITHMS}

The efficiency of the discussed self-calibration methods depends on the type of nonlinearity of the real conversion characteristic of measurement apparatus as well as on the number of interpolation knots (number of calibration points) used. The interpolation error for conversion characteristic is:

$$
\Delta=S_{A}(y) \cdot y-\varphi(y)
$$

As a comparison criterion we will use the limit error value:

$$
\Delta_{\max }=\max |\Delta| \text {. }
$$

The paper presents the comparison of self-calibration methods with linear in intervals and polynomial interpolation of conversion characteristics. The comparison was made for exemplary, typical measurement channels, containing the following converters in the apparatus: 'relative change of strain gauge bridge unbalance voltage' to frequency, conversion function of which is [2]:

$$
z=z_{0}+k_{1} \cdot y+\frac{k_{2}}{1+k_{3} \cdot y},
$$

where:

$z$ - frequency, $y$ - relative bridge unbalance voltage, $k_{1} \div k_{3}$ - parameters of measurement apparatus, as well as a dual-slope integrating $\mathrm{A} / \mathrm{D}$ converter with conversion function:

$$
z=k \cdot \ln \left[1+y \cdot\left(1-\mathrm{e}^{-k}\right)\right]
$$

where:

$Z$ - relative time (output time to integration time), $y$ - relative voltage (output to reference voltage),

$k$-aggregated parameter of $\mathrm{A} / \mathrm{D}$ converter.

The influence of the real values of electronic elements expressed by apparatus' parameters $k$ and $k_{1} \div k_{3}$ and their possible changes caused by disturbances were taken into consideration in these channels.

To exemplify the case, Fig. 3 shows the dependence of the limit error (16) on the number of calibration points (interpolation knots) for the converter 'relative change of strain gauge bridge unbalance voltage' to frequency (17), taking into consideration real properties of amplifiers (offset causing nonlinearity). Fig. 4 shows the dependence of the limit error (16) on the number of calibration points (interpolation knots) for the dual-slope A/D converter (18), taking into consideration dielectric loss of the integrating capacitor. The self-calibration methods used are linear in intervals interpolation of conversion function (curve 1 in the figures) and polynomial interpolation of conversion function (curve 2 in the figures).

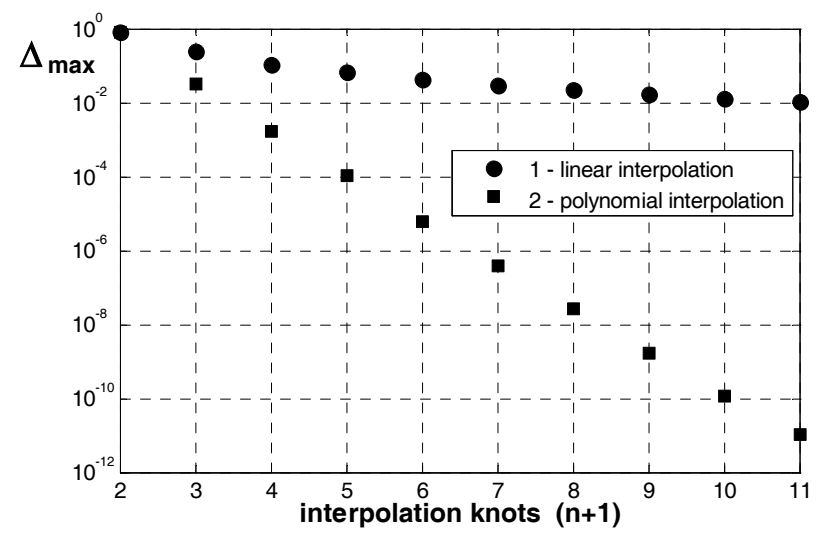

Fig. 3. Limit error (16) versus the number of calibration points for the converter 'bridge unbalance voltage' to frequency. 


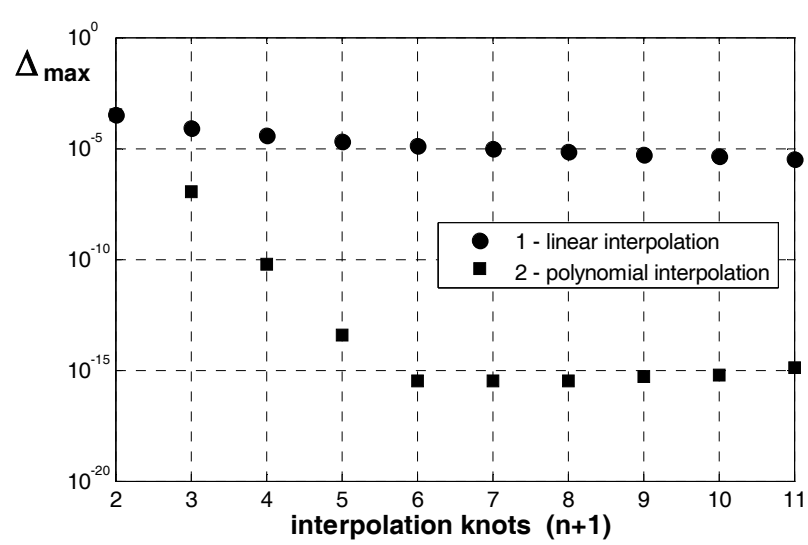

Fig. 4. Limit error (16) versus the number of calibration points for the dual-slope $\mathrm{A} / \mathrm{D}$ converter.

On the basis of the diagrams it may be ascertained that for the analyzed exemplary measurement channels the polynomial interpolation method shows a more rapid decrease of the limit error. For the two interpolation knots $(n=1)$, the effects of the methods efficiency agree, which is obvious; so, the values of the limit error (16) are identical. For three and higher number of interpolation knots, the error of polynomial interpolation decreases rapidly, giving satisfactory values by three knots. In the case of interpolation with function linear in intervals, the interpolation error decreases very slowly. For the example from Fig. 3, the error of linear interpolation decreases for two orders not before 11 interpolation knots, while for polynomial interpolation 3 knots are enough, as in the example from the Fig. 4. Delimiting further decrease of the error (Fig. 4) to the value of $10^{-15}$ is the result of limited exactness of calculations performed in the Matlab environment. When the self-calibration procedure is accomplished, the application of polynomial interpolation will enable us to delimit essentially the number of necessary reference sources (Fig. 2).

\section{REFERENCES}

[1] Gawędzki, W. (1996). Accuracy analysis of a measurement system auto-calibration method. In Proceedings of the IMEKO TC4: 8th International Symposium on New Measurement \& Calibration Methods of Electrical Quantities \& Instruments, 16-17 September 1996 (pp. 218-221). Budapest, Hungary.

[2] Gawędzki, W. (2006). Algorytm auto-kalibracji torów pomiarowych poprzez wielomianową interpolację funkcji przetwarzania (An algorithm of self-calibration for measurement channels by means of polynomial interpolation of conversion function). Pomiary, Automatyka, Kontrola 10 bis, 196-199.

[3] Gawędzki, W. (2006). Self-calibration methods in measurement channels for linear and non-linear conversion functions case. In Proceedings of the IEEE IMTC 2006, 24-27 April 2006 (pp. 1506-1510). Sorrento, Italy.

[4] CAMPBELL SCIENTIFIC (2001). Voltage measurement accuracy, self-calibration, and ratiometric measurements. Inc. Logan, Utah. Retrieved from http://www.campbellsci.ca/CampbellScientific/Download / VoltAccy.pdf

[5] CHenYang TECHNOLOGIES, Self-calibration measuring methods and systems. ChenYang Engineering Office ISM for Sensors Magnetics \& Measurements. Retrieved from (http://www.chenyang-ism.com/SC_Methods.htm).

[6] Liu, J.-G., Frühauf, U. (1999). Self-calibration measuring methods and application to measurements of electrical quantities. Measurement 26(2), 129-141. 